\title{
WATER DEMAND PREDICTION USING ARTIFICIAL NEURAL NETWORK FOR SUPERVISORY CONTROL
}

\author{
B. I. Gwaivangmin ${ }^{1, *}$ and J. D. Jiya ${ }^{2}$ \\ 1 Directorate of Physical Facilities, University of Jos, Jos, Plateau State. NIGERIA

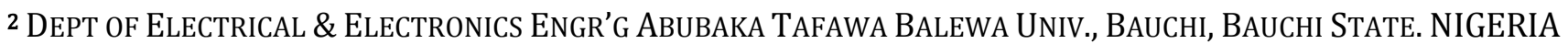 \\ E-mail addresses: ${ }^{1}$ bgwaivangmin@gmail.com, ${ }^{2}$ jibjiya27@gmail.com
}

\begin{abstract}
With increase in population growth, industrial development and economic activities over the years, water demand could not be met in a water distribution network. Thus, water demand forecasting becomes necessary at the demand nodes. This paper presents Hourly water demand prediction at the demand nodes of a water distribution network using NeuNet Pro 2.3 neural network software and the monitoring and control of water distribution using supervisory control. The case study is the Laminga Water Treatment Plant and its water distribution network, Jos. The proposed model will be developed based on historic records of water demand in the 15 selected demand nodes for 60 days, 24 hours run. The data set is categorized into two set, one for training the neural network and the other for testing, with a learning rate of 50 and hidden nodes of 10 of the neural network model. The prediction results revealed a satisfactory performance of the neural network prediction of the water demand. The predictions are then used for supervisory control to remotely control and monitor the hydraulic parameters of the water demand nodes. The practical application in the plant will cut down the cost of water production and even to a large extend provide optimal operation of the distribution networks solving the perennial problem of water scarcity in Jos.
\end{abstract}

Keywords: Water Demand, Prediction, Neural Network, Distribution, Scarcity

\section{INTRODUCTION}

Jos is a city in the middle belt of Nigeria, it has a population of about 900,000 residents based on the 2006 census. It is the administrative capital of plateau state. The city is located on the Jos Plateau at an elevation of about 1,238 meters or 4,062 feet high above sea level. The Laminga Treatment Plant presently is undersized considering the population growth in the past decades, more so that no expansion has been made since its commissioning in 1972. Due to its inability to meet up with present water demand, there is the need to forecast future water demand in order to forestall reoccurrence of water scarcity with government plan for intervention in the water sector.

Global demand for water is continuously increasing due to population growth, industrial development, and improvements of economic conditions, while accessible sources keep decreasing in number and capacity [16]. The quantities of water required for basic human needs are minimal. However, the fresh water that is available is under increasing pressure in many countries. According to [2], developing countries consume more water than the developed countries under the same condition.
The competition for the available water is expected to increase six and a half times over the next decades [19]. At the country level, it is suggested by [7] that the regular shortages of water (water stress) will occur when the annual renewable fresh water available per person falls below 1700 cubic meters. If availability falls below 1000 cubic meters per capital per year (water scarcity), health, economic development and human well being will be hampered. Considering water as a scarce resource requires proper planning and management, which requires good estimates of the water future demands.

According to [1], there are several factors known as drivers or explanatory variables affect the water demand forecast. These include socioeconomic parameters (population, population density, housing density, income, employment, water tariff, etc),weather data (temperature, precipitation, etc), as well as cultural factors such as consumer preferences and habits.[10] in their view said Selection and validation of any statistical models are very crucial in modeling and forecasting problems. In multiple regression analysis of forecasting long term water demand, various models are developed with a variety of predictor variables. Moreover, multiple 
regression models can take different forms such as linear, semi-log and log-log. In this paper, an effective but simple procedure named Monte Carlo cross validation (MCCV) is applied and compared to the most widely used leaveone-out validation(LOO) to select the best multiple regression model to forecast water demand. Unlike LOO validation, MCCV leaves out a major part of the sample during validation. Both methods are also used for estimating the prediction ability of the selected model on future samples. The advantage of MCCV is that it can reduce the risk of over fitting the model by avoiding an unnecessary large model. In this paper, MCCV and LOO are applied to the water demand data set for the Blue Mountains, NSW in Australia for single dwelling residential sector.

Various researchers have carried out forecasting studies in a number of ways in the past decade. According to [9], the total amount of water consumed must be balanced with the amount of water produced. To maintain this water balance between production and consumption one must appropriately forecast future water demand. Water demand forecast can be divided into three categories: short term- forecast, medium- term forecast and longterm forecast. The nature of these forecast are different as well. Short- term water demand forecasting can help to estimate water quantity and to make decisions that can prevent water scarcity. Timely implementation of such decisions lead to the improvement of network reliability and to the reduced occurrence of pipe burst and plant breakdown. On the other hand long- term forecasting helps to know the water demand in the future and take necessary actions, like the extensions of pipe networks, building of more treatment plants and reservoirs to fulfil the need of consumers. Statistically, forecasting is an approximation of future data. The water demand forecasting task is usually carried out by statistical methods with some adjustments by experienced operators in the various treatment plants. Traditional linear models, such as autoregressive (AR) and auto regressive moving average (ARMA), have been used in time series forecasts in practice. These models are straight forward for implementation, but there are limitations to improve forecasting accuracy by these methods which are constructed by linear functions. On the other hand, according to [20] an important contribution of Artificial Neural Networks (ANN) is their capability to do non-linear curve fitting. ANN models are particularly powerful when applied to a very complex data set and when the structure of the model is unknown. In [5], it was pointed out that in time series analysis the autoregressive integrate moving average (ARIMA) models have been used for decades and due to their success they have been used in a wide variety of scientific applications. In recent years a growing popularity of machine learning algorithms like the artificial neural network (ANN) and support vector machine (SVM) have led to new approaches in time series analysis. Especially the ability to consider nonlinearities gives these methods an advantage.[12] explored the use of neuro-genetic algorithm for daily water demand forecasting. [3], carried out the statistical methodology of Design of Experiments (DOE) was applied to better determine the parameters of an Artificial Neural Network (ANN) in a problem of nonlinear time series forecasting. Instead of the most common trial and error technique for the ANN's training, DOE was found to be a better methodology. According to[14],considering the increasing demand for the optimization of water distribution networks in terms of leakage reduction and pressure management, as well as the need to reduce urban water consumption, a lot of effort has been invested in the past decade in order to define accurate, long-term and short-term water demand forecasting methods. Linear regression models, such as ARIMA, and Artificial Neural Networks (ANN) have been used, as well as different hybrid approaches.[11] showed that artificial neural network is a powerful tool for input - output mapping and can be effectively used for reservoir inflow forecasting and operation. In an era when global water consumption is of increasing concern, forecasting water demand consumption is more important than ever.

Jos the plateau state capital in central Nigeria has been characterized with water scarcity. The city had its major treatment plant, the Laminga treatment plant built in 1972, and since then there has been no major expansion in the network being served by the plant. The growing population of the city has forced the plateau state water board into rationing water to consumers and this has led to high cost of potable water in the city. The need for forecasting the water demand in a water network cannot be over-emphasized. The population of these areas has almost doubled since the last expansion of the treatment plant. This has significant implications for the water distribution infrastructure in that area. The major source of water still remains the Laminga Treatment plant.

The objectives of this paper are to forecast water demand at the water demand nodes and to monitor and control the water distribution using supervisory control.

\section{MATERIALS AND METHODS}

The case study is the Laminga water treatment plant Jos and its distribution network, which covers old airport Road. Yakubu Gowon Way, Murtala Mohammed way, Dogon Karfe, Giring, Abattoir, mobile Barrack, Air Force Barracks, Gold and Base, Gwarandok, Daku, Rikkos, Liberty Boulevard, Apata, Zaria Bye-pass, Tafawa Balewa street, University of Jos, etc. 
The Laminga water distribution network contains 50 nodes and 5500 major pipes: it also has two reservoirs, old eastern site reservoir $-9200 \mathrm{~m}^{3}$ and ministry of works yard reservoir- $5500 \mathrm{~m}^{2}$ there are five fixed speed pumps; in which two operate on $600 \mathrm{~m}^{3} / \mathrm{h}$, and three on $341 \mathrm{~m}^{3} / \mathrm{h}$ that are used to pump water from the treatment plant to the reservoirs. Two pumps with the capacity of $341 \mathrm{~m}^{3} / \mathrm{h}$ are to be used to pump water to the ministry of works reservoir, with one pump on standby, while pumping to the old eastern site reservoir is done with one pump of capacity $600 \mathrm{~m}^{3} / \mathrm{h}$, with one on standby. But in practice three of the pumps for Ministry of works and two for the Eastern site are all put into use because it is believed it will pump more water to meet the water demand of the metropolis[17].

\section{RESULTS}

\subsection{Data Collection}

The forecast data for the research is 24 hours run of the Laminga water treatment plant and its water distribution network for 60 days at each of the water demand nodes. The historical record of Water demand for the 15 demand nodes, for 24 hours was obtained from the operations plant record book. These were used as input to the Back propagation Neural Network and the forecast (predicted) water demand.

\section{INTRODUCTION TO NEURAL NETWORK}

An artificial neuron is a computational model inspired in the natural neurons. Natural neurons receive signals through synapses located on the dendrites or membrane of the neuron. When the signals received are strong enough (surpass a certain threshold), the neuron is activated and emits a signal though the axon. This signal might be sent to another synapse, and might activate other neurons (Figure.1)

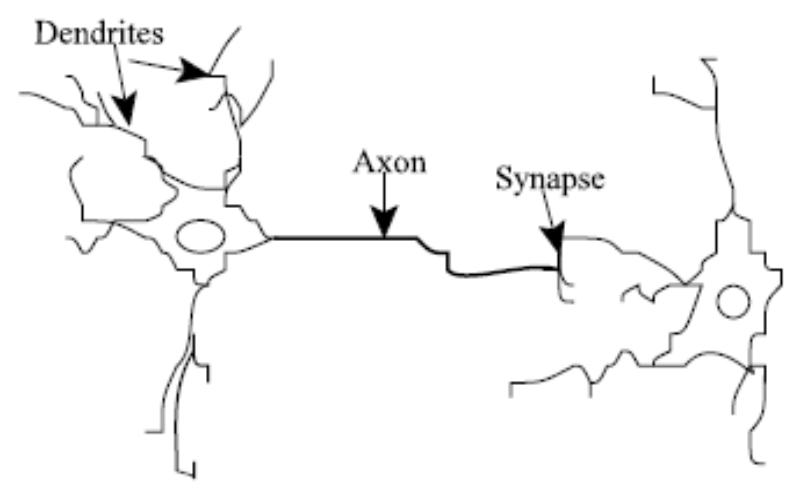

Figure 1: Natural Neurons (artist's conception).

The complexity of real neurons is highly abstracted when modelling artificial neurons. These basically consist of inputs(like synapses), which are multiplied by weights(strength of the respective signals), and then computed by a mathematical function which determines the activation of the neuron. Another function (which may be the identity) computes the output of the artificial neuron (sometimes in dependence of a certain threshold). ANNs combine artificial neurons in order to process information.

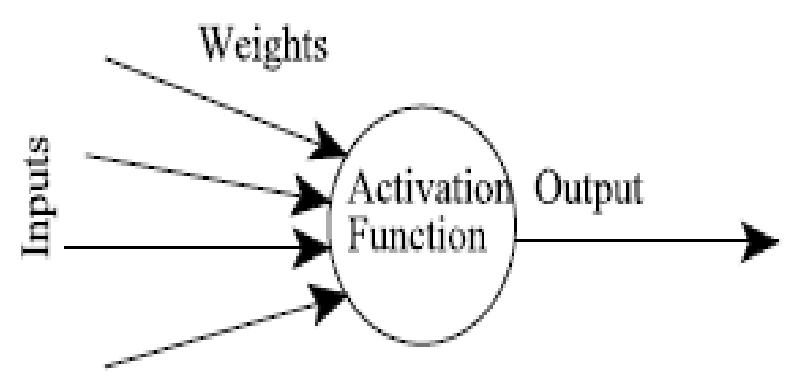

Figure 2:An Artificial Neuron

The higher a weight of an artificial neuron is (Figure 2), the stronger the input which is multiplied by it will be. Weights can also be negative, so we can say that the signal is inhibited by the negative weight. Depending on the weights, the computation of the neuron will be different. By adjusting the weights of an artificial neuron we can obtain the output we want for specific inputs. But when we have an ANN of hundreds or thousands of neurons, it would be quite complicated to find by hand all the necessary weights. But we can find algorithms which can adjust the weights of the ANN in order to obtain the desired output from the network. This process of adjusting the weights is called learning or training. The number of types of ANNs and their uses is very high. Since the first neural model by McCulloch and Pitts [13] there have been developed hundreds of different models considered as ANNs. The differences in them might be the functions, the accepted values, the topology, the learning algorithms, etc. Also there are many hybrid models where each neuron has more properties than the ones we are reviewing here. Because of matters of space, we will present only an ANN which learns using the back propagation algorithm for learning the appropriate weights, since it is one of the most common models used in ANNs, and many others are based on it. Since the function of ANNs is to process information, they are used mainly in fields related with it. There are a wide variety of ANNs that are used to model real neural networks, and study behaviour and control in animals and machines, but also there are ANNs which are used for engineering purposes, such as pattern recognition, forecasting, and data compression [8].

\subsection{Neural Network Prediction Model}

The objective of this model is to implement the mechanism and systems that can be employed to forecast 
both short - term and long - term water demand. The increasing growing field of computational intelligence technique, neural network, has been used as an efficient tool to forecast the water demand in the 15 water demand nodes.

The back propagation training algorithm could be employed to train the neural network. This is because it is efficient, easy to implement and does not consume time. The back propagation is a feed forward scheme that is interconnected by layers. If a set of inputs is applied to the input layer, it propagates through hidden layer until an output is generated from the output layer. The output is then compared with the desired output in which an error signal is computed for each output node. The error signals are then transmitted backwards from the output layer to each node in the hidden layers to update the connection weights. This process continuous until the error signal is as desired. The computation steps for the backpropagation algorithm are as follows:

- Initialize the interconnection weights and the node biases randomly.

- Calculate the hidden layer output as:

$$
x_{j}^{h}=f\left(\sum_{i=1}^{n_{i}}\left(x_{i} w_{i, j}^{h}\right)+b_{j}^{h} w_{n_{i}+1, j}^{h}\right)
$$

Where $x_{j}^{h}$ is the output of the hidden node $j, x_{i}$ is the $i t h$ input, $w_{i, j}^{h}$ is the weight connecting input node $i$ with hidden node $j, b_{j}^{h}$ is the input bias to hidden node $j$ (normally $b_{j}^{h}=1$ ), $w_{n_{i}+1, j}^{h}$ is the weight connecting the bias to the hidden node $j, n_{i}$ is the number of input nodes, and

$$
f(x)=\frac{1}{1+e^{-x}} \quad \text { (Sigmoid function) }
$$

- Calculate the outputs of the output layer nodes as:

$$
x_{k}^{o}=f\left(\sum_{i=1}^{n_{k}}\left(x_{j}^{h} w_{j, k}^{o}\right)+b_{k}^{o} w_{h_{i}+1, k}^{o}\right)
$$

Where $x_{k}^{o}$ is the output of the output node $k, w_{j, k}^{o}$ is the weight connecting the hidden node $j$ with output node $k, b_{k}^{o}$ is the input bias to the output node $k$ (normally $\left.b_{k}^{o}=1\right), w_{h_{i}+1, k}^{o} \mathrm{~s}$ the weight connecting the bias to the output node $k$, and $n_{h}$ is the number of hidden nodes.

- Calculate $\delta_{k}^{o}$ of each of the output nodes as:

$$
\delta_{k}^{o}=x_{k}^{o}\left(1-x_{k}^{o}\right)\left(x_{k}^{T}-x_{k}^{o}\right)
$$

Where $\delta_{k}^{o}$ is the error (target-output) at the output of the neuron multiplied by the derivative of $f(x), x_{k}^{T}$ is the target output (desired output) of the node $k$.

- Calculate $\delta_{j}^{h}$ of each of the hidden node as follows:

$$
\delta_{j}^{h}=x_{j}^{h}\left(1-x_{j}^{h}\right) \sum_{k=1}^{n_{o}} \delta_{k}^{o} w_{j, k}^{o}
$$

Where $\delta_{j}^{h}$ the derivative of is $f(x)$ multiplied by the summation of the weights multiplied by the output delta.

- Adapt the weights of the output layer as:

$$
w_{j, k}^{o}(t+1)=w_{j, k}^{o}+(t)+\mu \delta_{k}^{o} x_{j}^{h}+\alpha \Delta w_{j, k}^{o}(t)
$$

Where $0<\mu<1$ is the learning constant, $0<\alpha<1$ is the momentum constant, and

$$
\Delta w_{j, k}^{o}(t)=w_{j, k}^{o}(t)-w_{j, k}^{o}(t-1)
$$

- Adjust the weights of the hidden layer as:

$$
\Delta w_{i, j}^{h}(t+1)=w_{i, j}^{h}(t)+\mu \delta_{j}^{h} x_{i}+\alpha \Delta w_{i, j}^{h}(t)
$$

Where $0<\mu<1$ is the learning constant, $0<\alpha<1$ is the momentum constant, and

$$
\Delta w_{i, j}^{h}(t)=w_{i, j}^{h}(t)-\Delta w_{i, j}^{h}(t-1)
$$

- Repeat the above steps consecutively until the square of the error becomes less than a preset value (designer's choice), which is defined as:

$$
e=\sum_{k=1}^{n_{o}}\left(x_{k}^{T}-x_{k}^{o}\right)^{2}
$$

The structure of the neural network remains fixed during training and only the weights are adapted. These algorithms should be subject to a rigorous analysis in order to determine their advantages and disadvantages when compared with other model - based algorithm [18].

\subsection{Data normalization}

The data is normalized before being input to the ANN. The input vectors of the training data are normalized such that all the features are zero-mean and unit variance. The target values are normalized such that if the activation function is unipolar sigmoid, they are normalized to a value between 0 and 1 (since these are the minimum and maximum values of the activation function, and hence the output of the ANN), and if the activation function is Bipolar sigmoid or Tan hyperbolic, they are normalized to a value between -1 and 1 and 0 and $1 / 2 \pi \sigma$ when the activation function is RBF. The test data vector is again scaled by the same factors with which the training data was normalized. The output value from the ANN for this test vector is also scaled back with the same factor as the target values for the training data. In order to simplify the task of the neural network, the data set was normalized to $[0,1]$ range using equation (2).

$$
X_{n}=\frac{\mathrm{X}_{\mathrm{o}}-\mathrm{X}_{\min }}{X_{\min }-X_{\min }}
$$

In (11) $X_{n}$ is the normalized data point, $X_{\mathrm{o}}$ is the original data point, $X_{\min }$ and $X_{\max }$ are the global minimum and maximum values of the data set respectively. This is done 
in order to ensure that the training is faster and the chance of getting stuck in the local optima is reduced [15]

\subsection{Supervisory Control}

A supervisory control and data acquisition (SCADA) system is a widely distributed computerized system, primarily used to remotely control and monitor the condition of field- based assets from a central location. It defines the current state of the network in terms of control settings, flow rates, hydrostatic pressure, pump load, valve settings, plant incoming voltages etc. Either way, considerable reliance on experience and judgment of the skilled personnel in deciding which pumps/valves to operate and when, in order to ensure compliance with constraints and minimize energy cost [4].

The economic benefits of the supervisory control data acquisition of the Laminga Treatment plant water distribution networks can be estimated inspite of few available data. No direct cost reduction can be attributed to the system in the earlier stage with very little operating data to rely upon. Supervisory control and data acquisition system does not replace all the operating personnel, and energy savings will be hard to estimate during the early phase of the operation.

\section{SIMULATION RESULTS AND DISCUSSIONS}

\subsection{Prediction of Water Demand}

In this research the forecasting tool used to predict the Water demand of the areas covered by this investigation is the Neural Network software called the NeuNet Pro. Version 2.3 developed by Cormac Technologies [6]. The NeuNet Pro 2.3 uses a 3 layer Back propagation neural network with a bias of 1.0. Training begins with all weights set at random numbers. For each data record, the forecast (predicted) value is compared to the desired (actual) value and the weights are adjusted to move the prediction closer to the desired value. Many cycles are made through the entire set of training data with the weights being continually adjusted to produce more accurate forecasts (predictions). The data set used in this study is the 60 days, 24 hours water demand recorded for the 15 nodes in Jos metropolis. The data is manipulated in two forms, namely, 'normalization' and 'training'.

The Base demand for each of the 15 water demand nodes is shown in Table 1. The parameter used for the Back propagation Neural network is shown in Table. 2 The distribution of data point for the neural network used is shown in Table 3. Parameters obtained after training is shown in Table 4.

\subsection{Supervisory control}

The supervisory control was implemented using the simulated SCADA within the platform of visual basic
Software. In this investigation simulated computer software with the aid of visual Basic studio was used to demonstrate how a SCADA could be used for the hydraulic parameters in the 15 selected nodes. A program was written in Visual Basic, with the aid of Visual Studio to implement the supervisory control of the water distribution network. This includes modules such as:

- $\quad$ Logging in (username and password).

- Interface for activation and deactivation of valves.

- Interface demand node for activation and deactivation of pumps.

To access the interface as indicated Rikkos junction (node 1) was activated, control pumps 1 \&2 were activated to allow water to be pumped into the network. The water flow and pressure for the Rikkos junction were visualized for various pump combination. The pumps and the Active water demand node were then shut down. The demonstration showed that the entire treatment plant process could be done and monitored at the computer terminal as expected of a SCADA.

The data were set for training and testing as shown in Figure 3. The predicted water demand for the 15 water demand nodes was plotted using NeuNet Pro software and theplots for the training and prediction are shown in Figures 4 -7 for Rikkos village, Nassarawa and Tafawa Balewa Street respectively.

Table 1: The water base demand for the 15 nodes

\begin{tabular}{lll}
\hline S/No & Demand Node & Water Demand $\left(\mathrm{m}^{3}\right)$ \\
\hline 1 & Rikkos Junction & 966.4 \\
2 & Rikkos Village & 3311 \\
3 & Govt. Guest House & 956 \\
4 & Film corporation & \\
5 & Liberty Dam road & 1370 \\
6 & Nassarawa & 3840 \\
7 & Pump street & 2800 \\
8 & Old Bukuru Park & 3320 \\
9 & Ofudumi Street & 1800 \\
10 & SarkinMangu street & 2870 \\
11 & Abdulsalami Street & 1920 \\
12 & Ojukwu Street & 2217 \\
13 & Apata Street & 3600 \\
14 & Rwang Pam Stadium & 2230 \\
15 & TafawaBalewa Street & 3500 \\
\hline
\end{tabular}

Table 2: Parameters used in the Back propagation Neural Network (NeuNet Pro 2.3)

\begin{tabular}{lc}
\hline Parameter & Value \\
\hline Number of Hidden Nodes & 15 \\
Learn Rate & 50 \\
Momentum & 50 \\
Jog Weights & $\mathrm{Nil}$ \\
Verify Rate & 05 \\
\hline
\end{tabular}

Table 3: The distribution of data point

\begin{tabular}{cc}
\hline Data Set & Distribution of data points \\
\hline Training set & 1200 \\
Testing set & 1440 \\
\hline
\end{tabular}


Table 4: Parameters Obtained after training.

\begin{tabular}{cccccc}
\hline Demand Node & $\begin{array}{c}\text { Best Error } \\
(\%)\end{array}$ & $\begin{array}{c}\text { Current Error } \\
(\%)\end{array}$ & $\begin{array}{c}\text { Normalised RMS } \\
\text { Error(\%) }\end{array}$ & $\begin{array}{c}\text { Actual Error } \\
(\%)\end{array}$ & Cycles Completed \\
\hline Rikkos village & 0.03 & 0.03 & 2.90 & 118.34 & 60260 \\
Nassarawa & 0.06 & 0.06 & 0.68 & 46.35 & 90880 \\
Tafawa Balewa & 0.1 & 0.1 & 1.18 & 72.25 & 80790 \\
\hline
\end{tabular}

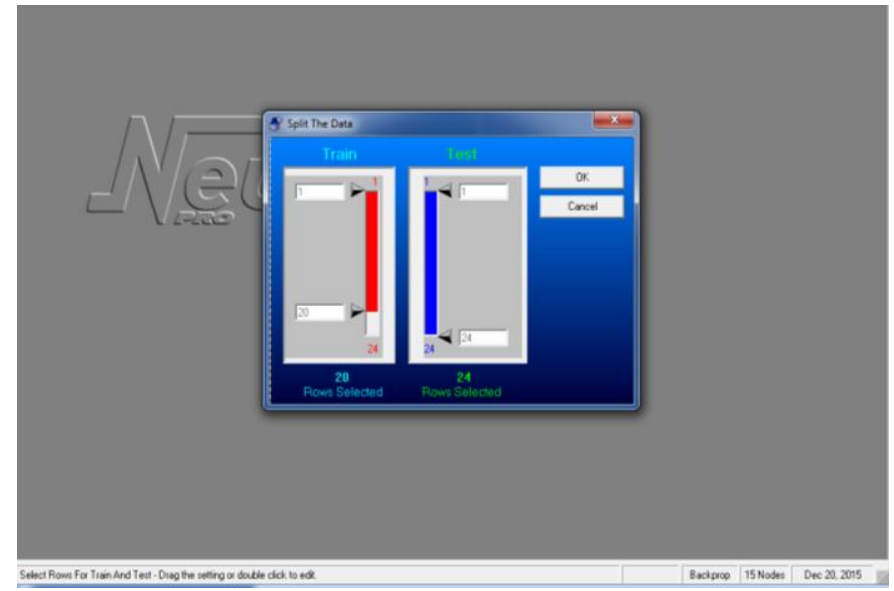

Figure 3: Setting for Training and Testing of Neural Network.

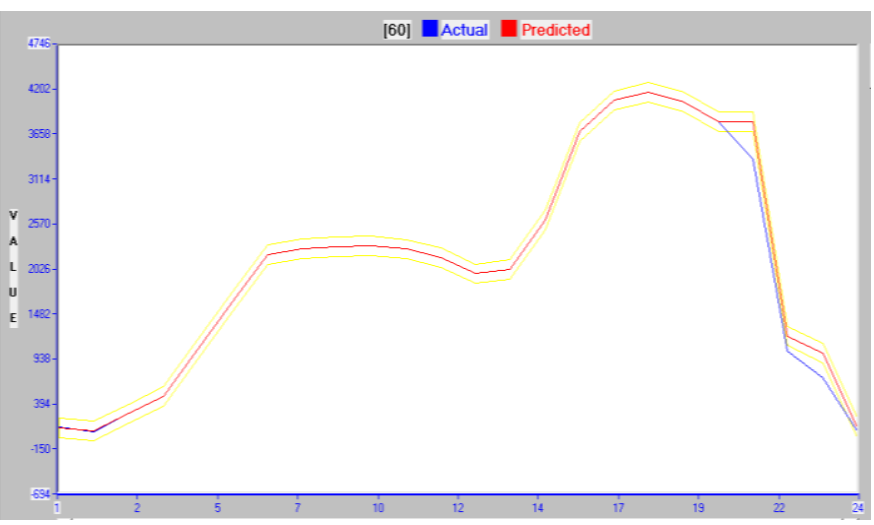

Figure 5: The Actual and Predicted Water Demand for Rikkos Village

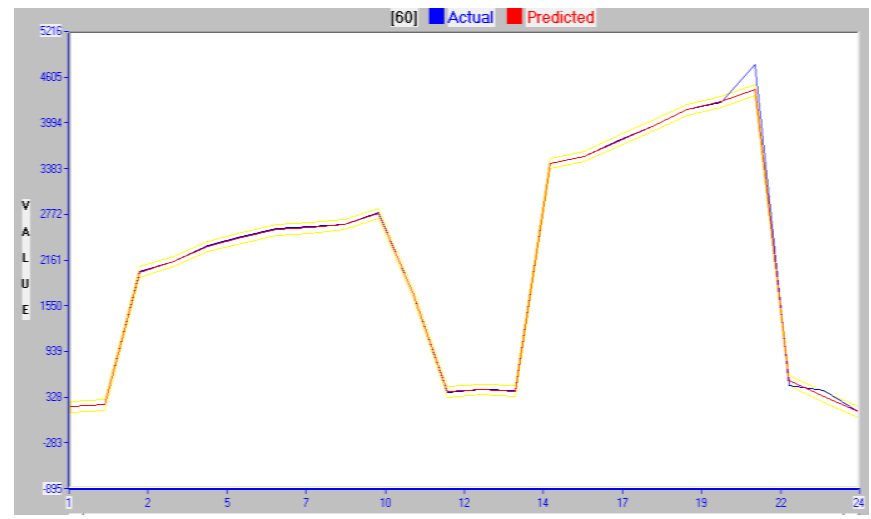

Figure 7: The Actual and Predicted Water Demand for Tafawa Balewa

\subsection{Discussion}

A number of similarity factors, influencing water consumption, were selected and used to come up with the input to our neural network; Such factors are the type of day (e.g. normal, public holiday, strike, major

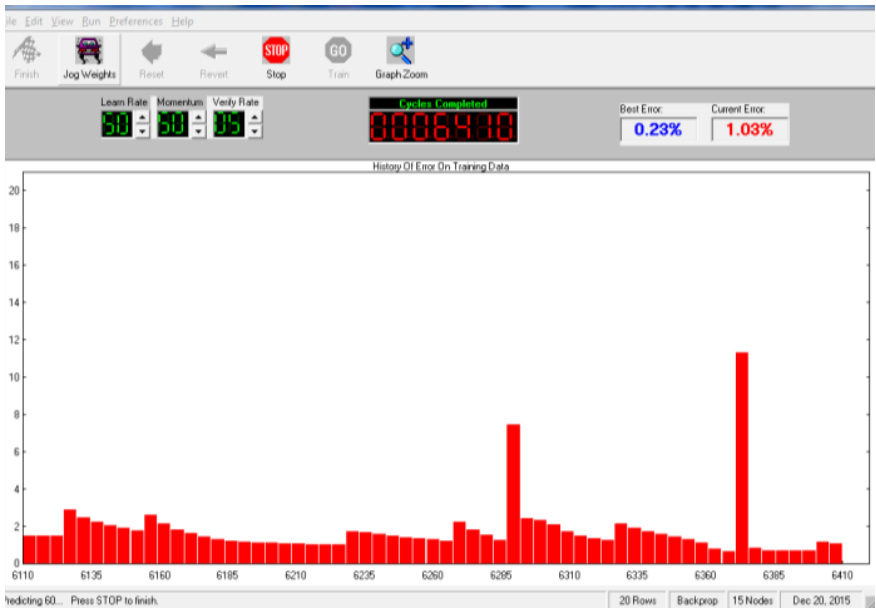

Figure 4.Training for Rikkos Village Water Demand

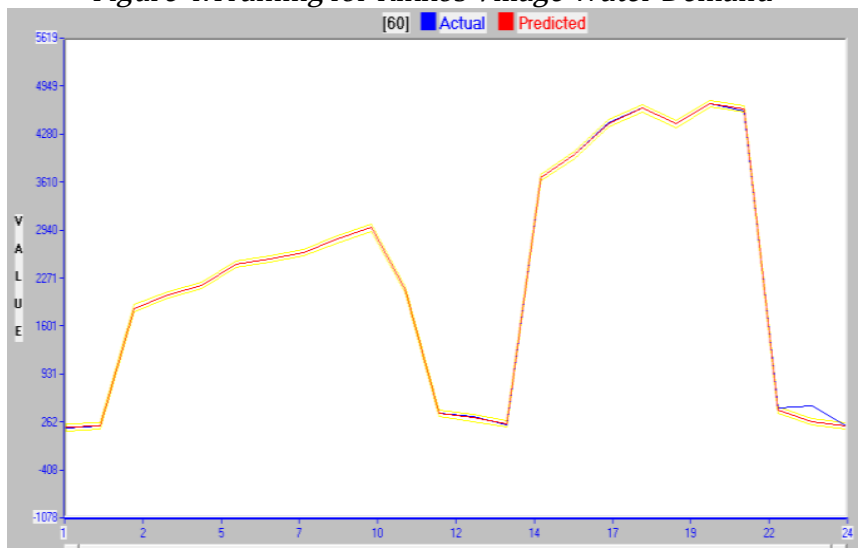

Figure 6: The Actual and Predicted Water Demand for Nassarawa

sports or other special event, etc.), day of the week, month, maximum and minimum temperatures, and the number of days after rainfall, population growth, and income of consumers to mention but few. This work focused on the importance of forecasting water demand for Laminga treatment plant in order to forestall future water scarcity presently affecting the water demand nodes. Supervisory Control was used to complement the forecasting work in order to achieve a considerable outcome, since the use of this facility is to help provide a fair water distribution in a situation of water scarcity. If the submission by Haestad et al. [9], that the total amount of water consumed must be balanced with the amount of water produced is strictly followed the issue of water scarcity will be reduced greatly. Ajbar and Ali [1] pointed out the several factors known as drivers or explanatory variables affect the water demand forecast. These were also considered in this paper. Haque et al. [10], said Selection and validation of any statistical 
models are very crucial in modeling and forecasting problems. Mellios et al [4], submitted that considering the increasing demand for the optimization of water distribution networks in terms of leakage reduction and pressure management, as well as the need to reduce urban water consumption, a lot of effort has been invested in the past decade in order to define accurate, long-term and short-term water demand forecasting methods. This work used the short term forecast of 24hours.

\section{CONCLUSION}

The Study showed each of the 15 water demand nodes predicted using the Back propagation neural network, with the aid of NeuNet Pro 2.3 software. The predicted water demand values of the demand nodes are useful for the next plant expansion project. If the forecast results are taken seriously there would no fear of water scarcity in the near future after the next plant expansion project. The Simulated computer software with the aid of Visual Basic studio used for supervisory control to monitor and control the water distribution in the network is a new development to enhance future plant operations. The Practical application in the plant will cut down the cost of Water production and even to a large extend optimal operation of the water distribution networks solving the perennial problem of water scarcity. The fact that to access the SCADA system, a password is required enhances the security of the entire treatment plant operations.

\section{RECOMMENDATIONS}

Forecasting water demand is very necessary for if there will be adequate water supply to consumers in our cities. Other forecasting methods are useful, but neural network is preferred because of its several advantages especially in the water supply industries where certain factors are not quantifiable and subject to error. Water utilities should be equipped with SCADA facilities to improve water distribution operations, cut down operational costs and enhance security. Fuzzy logic could be used alongside neural network to obtain better results.

\section{REFERENCES}

[1] Ajbar, A.H and Ali, E. " Water Demand Prediction for Touristic Mecca City in Saudi Arabia, Using Neural Networks", International Journal of Environmental, Chemical, Ecological, Geological and Geophysical Engineering. 6(5) pp 231-235, 2012.

[2] Al-layla, M. A. H., Shamin, A., and Middlebrooks, E. J. Water Supply Engineering Design, Ann Arbor Science Publishers, The University of Michigan paperback, 1978

[3] Balestrassi, P. P., Popova, E., Paivo, A. P and Marangon, L .J. W."Design of Experiments on Neural Network Training for nonlinear Time Series Forecasting", NeuroComputing,72 (1) pp.1160-1178, 2009.
[4] Bilmes, J. S ." SCADA and Network Analysis, Hydraulics and Hydrology in the Small Computer Age", American Society of Civil Engineers. (2) pp 880-886, 1985.

[5] Braun, M., Bernard, T., Piller, 0 and Sadehizade, F." 24 Hours Demand Forecasting Based on SARIMA and Support Vector Machine", $16^{\text {th }}$ Conference on Water Distribution System Analysis (WDSA) pp 926-933, 2014.

[6] Cormac Technologies. "NeuNet Pro 2.2."www.cormatech.com/neunet. Accessed on March 202015.

[7] Falkernmark, $M$ and Lindh, H."Water and Economic Development, Water in Crisis, a Guide to the World's Fresh Water Resources", P. H. Gleick, Oxford, Oxford University Press paperback,1993.

[8] Gershenson, C."Artificial Neural Network for Beginners, Neuro -evolutionary Computing Tutorial", Cornell University, arXiv. C.S/030803,2003

[9] Haestad., Walski, T. N.,Chase, D.V.,Savic, A. S., Grayman, W, Bechwith, and Koalle, E. Advanced Water Distribution Modelling and Management Heastad Methods inc. Waterbury. U.S.A, 2003.

[10] Haque, M. M., Hadda, K. ,Rahman, A. ,Hossain, M., Hagere, D and Kabria, G." Long Term Demand Forecasting: Use of Carlos Cross Validation for the Best Model Selection",20th International Congress on Modelling and Simulation, Adelaide, Australia, pp 2311-2317, 2013.

[11\} Jain .S. K., Das. A and Srivastava. D. K. "Application of ANN for Reservoir Inflow Prediction and Operation", Journal of Water Resources Planning and Management, 125(2)pp 263-271,1999.

[12] Joong, H. K., Seok, H. H and Hyun, S.S."An optimal Neural Network Model for Daily Water Demand Forecasting", WRPMD (99) pp1-10, 1999.

[13] McCulloch, W. and W. Pitts (1943). A Logical Calculus of the Ideas Immanent in Nervous Activity. Bulletin of Mathematical Biophysics, (5) pp. 115-133, 1943.

[14] Mellios, N., Kofinas, D. ,Papageorgiou, E and Laspidou, C," A Multivariate Analysis of the Daily Water Demand of Skiathos Island, Greece, Implementing the Artificial NeuroFuzzy inference System (ANFIS)",E-Proceedings of the $36^{\text {th }}$ IAHR World Congress, The Haque.pp1-9, 2015

[15] Arbib, M. A.The Handbook of Brain Theory and Neural Networks. A Bradford Book. The MIT Press, London, England, 2003.

[16] Mohammed, N.G.N, and Adulrahman, A." Water supply network system control base on Model Predictive Control", Proceedings of the Internal Multi-Conference of Engineering and Computer Scientists, IMECS, (2) pp11721177, 2009.

[17] PSWB. Plant Installation Manuals and Record Book, Plateau State Water Board (PSWB) Engineering Services, Plant Booklets, 1(1), 1972.

[18] Rojas, R . Neural Network, a Systematic Introduction. Springer, Verlag, Berlin. pp 421-436, 1996.

[19] Seralgeldin, I. Water Resources Management: A new Policy for Sustainable Future in Water Resources Development, International Journal of Water Resources Development (2)3 pp 221-232, 1995.

[20] Ungar, L H . Forecasting- The Handbook of Brain Theory and Neural Networks, Massachusetts Institute of Technology, the Mit Press Cambridge, Massachusetts, London, England,1995. 